\title{
Root vacuolar sequestration and suberization contribute to salinity tolerance in Pistacia spp. rootstocks
}

\author{
Shuxiao Zhang ${ }^{1}$, Alessandra Quartararo ${ }^{1}$, Oliver Betz ${ }^{1}$, Shahab Madahhosseini ${ }^{1}$, Angelo \\ Heringer $^{1}$, Thu Le ${ }^{1}$, Yuhang Shao ${ }^{1}$, Tiziano Caruso ${ }^{2}$, Louise Ferguson ${ }^{3}$, Judy Jernstedt ${ }^{1}$, \\ Thomas Wilkop ${ }^{1}$, and Georgia Drakakaki ${ }^{1}$ \\ ${ }^{1}$ University of California Davis \\ ${ }^{2}$ University of Palermo \\ ${ }^{3} \mathrm{UC}$ Davis
}

June 15,2020

\begin{abstract}
Pistachio (Pistacia spp.) is a tree nut crop with relatively high salinity tolerance. Currently, limited information exists on its rootstock's cellular responses to salinity stress, especially in its roots. In this study, we investigated salinity tolerance at cellular level, in two pistachio rootstocks, Pistacia integerrima (PGI) and a hybrid, P. atlantica x P. integerrima (UCB1). Root tip sections were categorized across a developmental gradient according to their xylem development, and their sodium content and suberin deposition were analyzed with fluorescence microscopy. Our data demonstrated a correlation between vacuolar sequestration of sodium ions $(\mathrm{Na}+)$ and salinity tolerance in the UCB1 genotype. In addition, UCB1 displayed higher basal levels of suberization in both the exodermis and endodermis that increased further after salinity stress. Notably, the root region immediately distal to the region of secondary xylem initiation showed the highest amount of vacuolar Na+ sequestration, indicating a developmental regulation of this process. Our cumulative data demonstrate that salinity tolerance in pistachio rootstock species is associated with both vacuolar $\mathrm{Na}+$ sequestration and suberin deposition at apoplastic barriers, and both are correlated with a root developmental gradient. These cellular characteristics are phenotypes that can be screened during the selection for salinity tolerant woody plant species.
\end{abstract}

\section{INTRODUCTION}

The combination of global climate change and dwindling freshwater supplies has increased the need for salt and drought tolerant crops. Among woody nut perennial crops, pistachio, a dioecious tree in the family Anacardiaceae, exhibits relatively high drought and salinity tolerance (Walker et al., 1987; Ahmad and Prasad, 2012). Thus, pistachio has emerged as a nut crop of increasing commercial interest in the USA and around the world (Ferguson et al., 2002; Karimi et al., 2009; Ahmad and Prasad, 2012). In laboratory and field conditions, thePistacia vera scions, grown on several Pistacia spp.rootstocks, can tolerate sodium chloride $(\mathrm{NaCl})$ concentrations of up to $150 \mathrm{mM}$ (Walker et al., 1987; Ferguson et al., 2002). In contrast, citrus, grape, and avocado are all characterized as relatively salinity sensitive tree crops, and grape and avocado can only tolerate up to $50 \mathrm{mM}$ and $15 \mathrm{mM} \mathrm{NaCl}$ respectively (Bernstein et al., 2004; Mohammadkhani et al., 2016; Ahmad and Anjum, 2020).

Salinity tolerance is a complex trait that involves the coordination of several interconnected mechanisms to minimize tissue damage upon salinity stress. These mechanisms include minimization of salt ion entry and loading into the xylem, maximizing intracellular compartmentalization in tissue, and ion retrieval before it reaches the leaves and shoot tips (Tester and Davenport, 2003; Gonzalez et al., 2012; Gupta and Huang, 2014; Chen et al., 2018; Yang and Guo, 2018; Munns et al., 2020). One well-studied mechanism is the regulation 
of ion homeostasis via the Salt Overly Sensitive (SOS) pathway at the plasma membrane. The SOS1, an $\mathrm{H}^{+} / \mathrm{Na}^{+}$antiporter, regulates $\mathrm{Na}^{+}$efflux from the cytosol to the surrounding extracellular medium, and has been associated with salinity tolerance phenotypes in Arabidopsis and halophytes, a group of plants known for their ability to survive in high salinity environments (Shi et al., 2002; Yang et al., 2009; Ji et al., 2013; van Zelm et al., 2020). The SOS pathway may also coordinate signaling with other salinity stress response pathways, such as the high affinity potassium channel (HKT ) located at the plasma membrane, to maintain ionic homeostasis by mediating potassium $\left(\mathrm{K}^{+}\right)$and $\mathrm{Na}^{+}$flux (Yang and Guo, 2018). The regulation of these pathways, for example HKT1, is considered one of the salinity tolerance determinants in several diverse species including Arabidopsis, grape, and potato (Henderson et al., 2018; Yang and Guo, 2018; Zhang et al., 2019).

Plants can maximize intracellular compartmentalization of salt in tissues by sequestering excessive salt ions within vacuoles to reduce cytosolic toxicity (Zhang and Blumwald, 2001; Gonzalez et al., 2012; Gupta and Huang, 2014; Munns et al., 2016; Guo et al., 2020). This process is mediated by the $\mathrm{H}^{+} / \mathrm{Na}^{+}$antiporters, encoded by the NHX1/2 genes (Zhang and Blumwald, 2001; Bassil et al., 2019). Additional layers of complexity include post-translational modification of antiporter proteins and retention of ions in the vacuole, both of which are associated with salinity tolerance (Tester and Davenport, 2003; Munns et al., 2016; Wu et al., 2019). There are limited studies in woody nut perennial species that investigate salinity stress responses at the cellular level with structural analysis and imaging methodologies. However, evidence is emerging in citrus for a role of vacuolar sodium sequestration in decreasing toxicity and minimizing sodium transported to leaves (Storey and Walker, 1998; Gonzalez et al., 2012).

Plants can also minimize salt entry via the regulation of ionic uptake through blockage of apoplastic transport. Specifically, apoplastic barriers can block bypass flow, which would otherwise allow $\mathrm{Na}^{+}$to enter the shoot through the transpiration stream (Chen et al., 2018). The endodermis and exodermis cellular barriers are the innermost and outermost layers of the root cortex, respectively (Enstone et al., 2003; Barberon, 2017). Their function as apoplastic barriers has been attributed to two notable features of their cell walls: 1) the Casparian strip and 2) the suberin lamellae (Enstone et al., 2003; Doblas et al., 2017). The Casparian strip diffusion barrier is a deposition of lignin along the radial and transverse cell walls of the endodermis and exodermis. It forms a hydrophobic apoplastic barrier forces all apoplastic transport into the more tightly regulated symplastic system (Naseer et al., 2012; Lee et al., 2013). In contrast, suberin lamellae, composed mainly of long chain fatty acids, impregnate the entire exodermis/endodermis cell wall (radial, transverse, and tangential), forming a hydrophobic barrier that may play a role in further regulating ion and water uptake (Enstone et al., 2003; Serra et al., 2009).

Salinity influences both the timing and extent of suberization in apoplastic barriers, which in turn can affect the entrance of salt into vascular tissue (Enstone et al., 2003; Chen et al., 2011; Byrt et al., 2018; Wang et al., 2020). Apoplastic barriers can control $\mathrm{Na}^{+}$uptake and transport to shoots, as demonstrated in rice, where blockage of apoplastic transport using silicate reduced sodium uptake (Yeo et al., 1999). Inhibition of suberin biosynthesis, via knockdown of CYP86A1/HORST, a cytochrome P450 dependent fatty acid $\omega$-hydroxylase, leads to increased levels of $\mathrm{Na}^{+}$concentrations in Arabidopsis shoots and roots (Wang et al., 2020). To date, there have been very few investigations regarding the development of apoplastic barriers in woody fruit and nut crop species. In citrus, preliminary studies indicate that suberin deposition may increase in response to salt in higher order roots, and higher suberin deposition in exodermis may be associated with lower sodium uptake (Rewald et al., 2012; Ruiz et al., 2016). Olive shows increased suberization in response to drought stress, which reduced root hydraulics (Tataranni et al., 2015). In Pistacia species, the contribution of apoplastic barriers to salinity tolerance has hitherto not been examined.

Studies on the effects of salinity on pistachio rootstocks have proposed multiple mechanisms that can contribute to salinity tolerance, such as rootstock ion sequestration, increasing osmolyte concentration in shoot and root, and sodium interception by xylem tissue, (Picchioni et al., 1990; Ferguson et al., 2002; Akbari et al., 2018; Rahneshan et al., 2018; Godfrey et al., 2019; Jamshidi Goharrizi et al., 2020). Sodium exclusion in stem vascular tissue, in combination with sequestration in roots, was postulated to decrease sodium trans- 
port to the leaves (Godfrey et al., 2019). Thus, a combination of multiple mechanisms working in tandem is likely to account for pistachio salinity tolerance.

Among the few studies focusing on salt tolerance in the rootstocks of woody perennial nut species, none have focused on fine roots, where the bulk of water and ion uptake occur, or in the root tips, where ions such as potassium may accumulate (McCully, 1995; McCully, 1999; Ranathunge et al., 2011). We used fluorescence microscopy to investigate the localization of sodium and the differentiation of the root endodermis and exodermis across a developmental gradient in salt treated pistachio rootstocks. Our results indicate that a combination of sodium sequestration and apoplastic barrier differentiation contribute to salinity tolerance in pistachio rootstocks, and that these responses are coordinated across a root developmental gradient.

\section{MATERIAL \& METHODS}

\section{Plant material and growing conditions}

P. integerrima and UCB1 seeds were provided by Foundation Plant Services, University of California (Davis, CA, USA), the Kresha Agricultural Nursery (Bakersfield, CA, USA) and from the Wolfskill Experimental Orchard (Winters, CA, USA).

Pistachio seeds were separated from shells and sterilized in a mixture of $5 \%(\mathrm{~V} / \mathrm{V})$ bleach and $1 \%(\mathrm{~V} / \mathrm{V})$ Tween-20 in deionized water. Seeds were germinated in vitro in $\frac{1}{2}$ Murashige-Skoog (MS) medium at adjusted $\mathrm{pH}$ of 5.7 containing $1 \%(\mathrm{~W} / \mathrm{V})$ sucrose, $0.1 \%(\mathrm{~W} / \mathrm{V})$ activated charcoal, and $0.75 \%(\mathrm{w} / \mathrm{v})$ agarose. The media were supplemented with $4.5 \mu \mathrm{M}$ 6-Benzylaminopurine, $0.5 \mu \mathrm{M}$ indole-3-butyric acid, $0.29 \mu \mathrm{M}$ gibberellic acid. Germinated seedlings were grown for 8 weeks for UCB1 and 10 weeks for P. integerrima in the same medium, at $22 \pm 2^{\circ} \mathrm{C}$ using fluorescent light $\left(100\right.$ to $150 \mathrm{mmol}$ quanta PAR $\left.\mathrm{m}^{-2} \mathrm{~s}^{-1}\right)$ under long day conditions (16 $\mathrm{h}: 8 \mathrm{~h}$ light : dark). Plants were transferred to charcoal-free supplemented $\frac{1}{2}$ MS medium for a minimum of one week prior to the start of the experiment.

\section{Salinity treatment}

Seedlings were transferred to a fresh charcoal-free supplemented $\frac{1}{2}$ MS medium or the same medium containing $100 \mathrm{mM}$ of sodium chloride. The plants were grown for one week, under the same conditions described above, before sectioning and further processing. Images of the plants were collected at the start and the end of the treatment for further phenotypic assessment.

\section{Sample collection}

During sample collection, seedlings were imaged, removed from medium, and the roots carefully rinsed to remove residual media. Excess water was removed by careful dabbing with Kimwipes (Thermo Fischer, 34120 ), and the length and weights of roots and shoots were measured.

\section{Salt ion analysis}

The roots from treated and control seedlings were carefully washed twice with deionized water and then dried for $24 \mathrm{~h}$ at $70^{\circ} \mathrm{C}$. Plant tissues were weighed and sealed in envelopes, then packed with desiccant to avoid hydration prior to ion analysis. Ion analysis was performed using inductively coupled plasma-mass spectrometry at the Baxter Laboratory Ionomoics Facility, Donald Danforth Plant Science Center (St. Louise, MO, USA) (https://www.baxterlab.org ) using established procedures (Ramirez-Flores et al., 2017).

\section{Fluorescent staining and microscopy}

Plant root tips from all shoot phenotype groups were cut into $0.5 \mathrm{~cm}$ segments starting from the distal tip. Root tip segments were embedded in $5 \%(\mathrm{~W} / \mathrm{V})$ agarose in deionized water and sectioned to 100 $\mu \mathrm{m}$ thickness using vibratome (Vibratome 1000 Plus Sectioning System) as previously described (Pradhan Mitra and Loqué, 2014). The sections were transferred into CoroNa Green incubation buffer (20 mM 3-(Nmorpholino)propanesulfonic acid, $0.5 \mathrm{mM}$ calcium sulfate, $200 \mathrm{mM}$ sorbitol). For CoroNa Green staining, 50 $\mu \mathrm{g}$ of CoroNa Green reagent (Thermo Fischer, C36676) was resuspended in $100 \mu \mathrm{L}$ dimethyl sulfoxide, and this solution was further diluted in incubation buffer to make $0.1 \mathrm{mM}$ staining solution. Sections were either 
incubated in staining solution or incubation buffer (as controls) for $16 \mathrm{~h}$ in the dark at room temperature before imaging. SNARF-1 staining (Thermo Fisher, C1270) was performed using a protocol adapted from Rosquete et al, 2019. Briefly, $1 \mu \mathrm{L}$ of $10 \mathrm{mM}$ SNARF-1 was diluted in $1 \mathrm{~mL}$ of CoroNa Green incubation buffer, and sections are incubated in this solution in the dark for $3 \mathrm{~h}$ at room temperature before imaging (Rosquete et al., 2019). Suberin staining was performed using a protocol adapted from Naseer et al (Naseer et al., 2012). Briefly, sections were incubated in $0.1 \mathrm{mg} / \mathrm{mL}$ Fluorol Yellow 088 (Santa Cruz Biotechnology CAS-81-37-8) in lactic acid for $1 \mathrm{~h}$ at $70{ }^{\circ} \mathrm{C}$ in the dark, rinsed in double deionized water, and imaged while mounted in 50\% glycerol. FDA staining (Sigma, \#F7378) was performed using protocol adapted from Jones et al (Jones et al., 2016). Briefly, live root sections were incubated in $4 \mu \mathrm{g} / \mathrm{mL}$ of FDA in incubation buffer for $5 \mathrm{~min}$ in the dark and imaged within $30 \mathrm{~min}$.

\section{Image acquisition and analysis}

A Zeiss 700 laser scanning confocal microscope was used for all analyses. Fluorescence signals of CoroNa Green (excitation $488 \mathrm{~nm}, 5 \%$ power, $493 \mathrm{~nm}$ emission filter) and CoroNa Green and SNARF-1 co-stain (excitation $488 \mathrm{~nm}, 15 \%$ power with $555 \mathrm{~nm}$ emission filter, $555 \mathrm{~nm}, 20 \%$ power with $566 \mathrm{~nm}$ emission filter) were collected with 20x objectives in CoroNa Green incubation buffer. Fluorescent signals of suberin (excitation $488 \mathrm{~nm}, 5 \%$ power, $493 \mathrm{~nm}$ emission filter) were collected with 20x object in $50 \%(\mathrm{~V} / \mathrm{V})$ glycerol in deionized water.

Quantification was performed using ImageJ software (Schneider et al., 2012). Briefly, the microscopy images were separated into groups based on xylem development. For each CoroNa Green sample imaged, a 3D maximum projection was assembled from z-stack using the Zen Black (Zeiss) software and exported as a single image file to Image $\mathrm{J}$, where $\mathrm{Na}^{+}$positive vacuoles were counted and recorded. The same export settings were used for all images of an experiment. For Fluorol Yellow 088 fluorescence quantification, lines were drawn through cell layer perpendicular to the radial cell walls of export 3D maximum projection images using the segmented line tool in ImageJ, and the signal intensity values along the line calculated by ImageJ. The values corresponding to the radial cell walls were used to obtain the average fluorescence value for the entire endodermis / exodermis cell layer in each section. For suberized cell count, the total number of cells and the total number of suberized cells in endodermis / exodermis were counted in ImageJ. The images used in figures were exported with Zen Black as described above and assembled using Illustrator

\section{Statistical analysis}

All statistical analyses were performed using R(R Core Team, 2017). All graphs are generated using Microsoft Excel.

\section{RESULTS}

Phenotypic characterization of leaf tissue showed higher percentage of salinity tolerant plants in UCB1 compared to $P$. integerrima

Pistachia integerrima (also known as PGI) and the hybrid UCB1 (P. atlantica $\mathrm{x}$ P. integerrima) are popular commercial rootstocks with P. vera scion cultivars (Zohary and Spiegl-Roy, 1975; Holtz et al., 2005). Earlier studies demonstrated the superior salinity tolerance of UCB1 rootstock compared to its paternal genotype $P$. integerrima (Ferguson et al., 2002). In order to better understand the origin of their different salt tolerances, we assessed the variation within and between UCB1 and $P$. integerrimaseedlings. Individual plants were treated with $100 \mathrm{mM} \mathrm{NaCl}$ for 7 days and compared to $0 \mathrm{mM}$ control plants of identical age (Fig. 1a). Salt tolerance was qualitatively assessed by the severity of leaf senescence or burn symptoms. Plant phenotypic classification after salt treatment was divided into three categories: 1) "high tolerance" when their leaf phenotype was indistinguishable from those of untreated plants, 2) "low tolerance" when over $75 \%$ of their leaves showed burns and / or senescence, 3) "moderate tolerance" when plants had an intermediate phenotype between that of "high tolerance" and "low tolerance". Based on this phenotypic characterization, over $40 \%$ of UCB1 plants demonstrated high tolerance for salinity, which is approximately twice the rate observed for P. integerrima (Fig. 1b). The dioecious nature of wind pollinated pistachio results in a heterogeneity of 
the seedlings (Ahmad et al., 2005), which likely contributed to the observed variation. Together, these data demonstrated that the UCB1 genotype has a higher percentage of plants with leaf traits indicative of high salinity tolerance, corroborating previous studies (Ferguson et al., 2002). The observed differences between $\mathrm{UCB1}$ and P. integerrimawere further explored to dissect their respective mechanisms of salinity tolerance.

\section{Short term salt treatment does not affect pistachio growth}

Next, we examined the roots and shoots of salt treated plants for developmental changes. It is known that UCB1 plants show more robust growth, potentially as a result of hybrid vigor. Hence, in order to avoid size effects on salinity tolerance and sequestration, we used 8 weeks old UCB1 plants and 10 weeks old $P$. integerrima plants for all our assays. At these ages, the two genotypes have a virtually identical fresh weight (Fig. 2a, NS, two-way ANOVA) After one week of salt treatment, neither P. integerrima nor UCB1 showed significant decreases in root or shoot growth (Fig. 2a, NS, two-way ANOVA, Suppl. Fig 1a).

Since root system architecture remodeling is known to occur as a result of salinity stress (Koevoets et al., 2016), we assessed the root architecture and root weight of the treated plants. Although the root weight of UCB1 and $P$. integerrima were comparable (Fig. 2a), the UCB1 plants had more lateral roots compared to $P$. integerrima, and this difference was further enhanced after salt treatment (Fig. $2 \mathrm{~b}, \mathrm{P}<0.05$ between genotypes, $\mathrm{P}<0.01$ between treatment, two-way ANOVA, $\mathrm{P}=0.01$ Tukey's HSD). No significant difference in lateral root lengths between the two genotypes was observed (Supp. Fig. 1b, NS, two-way ANOVA). In Arabidopsis accession Columbia-0, low concentrations of salt have been shown to induce more lateral root initiation, possibly due to an enhanced cell cycle activity and cell elongation through increased $\mathrm{Na}^{+}$vacuolar sequestration (Julkowska et al., 2014). Our results show that similar responses are occurring inPistacia spp. after short term salt treatment.

\section{UCB1 shows vacuolar $\mathrm{Na}+$ sequestration}

In order to investigate the mechanism of salt tolerance, we visualized the cellular localization of sodium ions in live root sections with CoroNa Green, an indicator that exhibits an increase in green fluorescence emission intensity upon $\mathrm{Na}+$ binding (Park et al., 2009; Gonzalez et al., 2012). Root developmental signals have several control points, and biotic stress has been shown to induce root differentiation closer to the apical meristem (Rost, 2011; Cajero-Sanchez et al., 2019). To assess the relationship between root maturity and salinity tolerance responses, $\mathrm{Na}^{+}$localization was analyzed across a root developmental gradient, staged by type and extent of xylem formation (Fig 3a, b, Supp. Fig. 2). The characterization of the stages was established as follows: zone 0 represented the youngest (least mature) differentiated root region in which only protoxylem is present, zone 1 represented the region of intermediate development in which both protoxylem and metaxylem are present, zone 2 represented the most mature region, in which development of secondary xylem has been initiated (Fig. 3b, Supp. Fig. 2).

We observed sodium localization in the cortical parenchyma cell vacuoles of salt treated UCB1 seedlings (Fig. 3a). We confirmed the viability and physiological activeness of the cells in the root cross sections with fluorescein acetate staining, which measures both enzymatic activity (required to activate its fluorescence) and cell-membrane integrity (required for intracellular retention of their fluorescent product) (Supp. Fig. 3). Comparison of vacuolar staining across all developmental zones showed a consistent increase in $\mathrm{Na}^{+}$ positive vacuoles in UCB1 compared to $P$. integerrima (Fig. 3c, $\mathrm{P}<0.01$ between genotype, $\mathrm{P}<0.05$, between treatment, two-way ANOVA). A post-hoc Tukey's HSD analysis verified the significant difference between control and treated UCB1 plants (Fig. 3c, $\mathrm{P}<0.05$ ), and showed no difference in $P$. integerrimaseedlings (Fig. 3c, NS, two-way ANOVA). Notably, the highest number of $\mathrm{Na}^{+}$stained vacuoles was observed in zone 1 of the UCB1 salt treated plants (Fig. 3d). This indicates an increase in $\mathrm{Na}^{+}$sequestration under salt stress in the active growth zones of the root tips, before the onset of secondary growth. In contrast, $P$. integerrima showed minimal vacuolar localization of sodium with no significant increase after salinity treatment (Fig 3a, c, d). This suggests that root tip sequestration of $\mathrm{Na}^{+}$is likely a trait specific to UCB1. The majority of the cortical parenchyma cells of both untreated UCB1 and $P$. integerrima plants did not stain positive for Na+ (Fig. 3a,c,d) demonstrating that $\mathrm{Na}+$ vacuolar sequestration was enhanced in salinity stress. 
Co-localization with the established vacuolar red fluorescent marker SNARF-1 (Rosquete et al., 2019) demonstrated that CoroNa Green indeed exhibited vacuolar localization (Fig. 4a-c). Negative controls did not show cross talk between the acquisition spectra of the dyes (Fig. 4d-i), confirming signal specificity and true vacuolar $\mathrm{Na}^{+}$localization. Furthermore, CoroNa-Green and SNARF-1 signal were not affected by plant tissue autofluorescence, confirming the validity of our imaging methodology (Supp. Fig. 4).

Since the vacuolar localization of $\mathrm{Na}^{+}$suggests sequestration in root tissue, we measured the $\mathrm{Na}^{+}$concentration in the roots of $P$. integerrima and UCB1 after one week of salt treatment. Although both UCB1 and $P$. integerrimaroots showed a significant $\mathrm{Na}^{+}$increase after $\mathrm{NaCl}$ treatment (Supp. Fig. 5, two-way ANOVA, $\mathrm{P}<0.01$ between treatment), the increase in UCB1 was significantly lower than that in P. integerrima (Supp. Fig. 5, two-way ANOVA, $\mathrm{P}=0.01$ ). This corroborates earlier studies that UCB1 has lower $\mathrm{Na}^{+}$concentration in aerial tissues compared to $P$. integerrima(Ferguson et al., 2002). We also measured the $\mathrm{K}^{+}$concentration, since it is an ion that is known to accumulate during salt stress as a result of the plant's need to maintain ionic homeostasis (Chen et al., 2018; Ahmad and Anjum, 2020). We did not observe significant changes in the $\mathrm{K}^{+}$concentration in roots of either UCB1 or P. integerrima after treatment.

Cumulatively, our data demonstrated increased sodium sequestration in the vacuoles of UCB1 roots compared to $P$ integerrima in response to salt treatment. However, the root $\mathrm{Na}^{+}$concentration was higher in $P$. integerrima, suggesting higher uptake and accumulation of non-vacuolar $\mathrm{Na}^{+}$in this salt sensitive genotype.

\section{UCB1 shows increased suberin deposition at the endodermis and exodermis}

In addition to $\mathrm{Na}^{+}$vacuolar localization, we observed a slight increase in $\mathrm{Na}^{+}$signal in the cell walls of the endodermis and the exodermis of the salt treated plants (Fig. 3a). Therefore, we investigated further whether the development of suberin lamellae in these two cell layers is regulated as part of the salinity stress response. We used a well-established methodology for suberin detection via staining with the lipophilic fluorochrome Fluorol Yellow (Lux et al., 2005; Kreszies et al., 2019) to quantify suberin deposition in the exodermis and endodermis.

Higher suberin deposition was observed throughout in the apoplastic barriers of UCB1 compared to $P$. integerrima, a pattern that was maintained or emphasized under salt treatment (Fig. 5a). Of the two barriers, UCB1 endodermis suberization showed a greater increase in response to salt treatment than $P$. integerrima, though the increase is statistically significant for both genotypes (Fig. 5b $\mathrm{P}<0.05$ for $P$. integerrima, $\mathrm{P}<0.01$ for UCB1, two-tailed t-test). In contrast, the exodermis of $P$. integerrimashows a significant increase in suberization to salt treatment that was not observed in UCB1 (Fig. 5b, $\mathrm{P}<0.05$ for $P$. integerrima, NS for UCB1, two-tailed t-test). When we examined these changes by developmental zones, we observed that in $P$. integerrima, the only statistically significant increase in suberization occurred in the exodermis of zone 1 (Fig. $5 \mathrm{c}, \mathrm{P}<0.05$, two-tailed t-test). Interestingly, the increase in suberization observed in UCB1 endodermis was statistically significant in both zones 0 and zone 1 , with the greatest difference observed in zone 0 (Fig. 5 d, $\mathrm{P}<0.01$ for zone $0, \mathrm{P}<0.05$ for zone 1 , two-tailed t-test).

We then compared the zone-specific responses between genotypes, in salt and control treatments. In control conditions, we found the greatest difference in zone 0 (Fig. 5e, $\mathrm{P}<0.01$ ), which is the youngest region possessing high developmental plasticity. While there was no significant difference between UCB1 and $P$. integerrimasuberization in zones 1 and zone 2 of control plants, under salinity stress all developmental zones showed significantly higher suberin deposition in UCB1 compared to $P$. integerrima (Fig. 5f, $\mathrm{P}<0.01$ for zone $0,<0.05$ for zone 1 and 2 , two-tailed t-test). This suggests a greater salt response and higher developmental plasticity of cellular barriers in UCB1, which would enable better control of apoplastic transport in all developmental zones.

In order to further investigate the distinction between suberin deposition and endodermis differentiation in the two genotypes, we quantified the percentage of suberized cells in the exodermis and the endodermis. While UCB1 showed a significant increase in the number of cells suberized after salt treatment in both endodermis and exodermis of $(\mathrm{P}<0.05$ for exodermis and endodermis, two-tailed t-test), P. integerrima only showed a significant increase in the endodermis $(\mathrm{P}<0.05$, two-tailed t-test). Further analysis revealed that 
there is a significant difference between genotypes in the percentage of cells suberized in the endodermis, but not the exodermis $(\mathrm{P}<0.01$ between genotypes, $\mathrm{P}<0.001$ between treatment, two-way ANOVA). UCB1 contained a reduced number of suberized cells in the endodermis layer compared to P. integerrima. This shows that the higher suberin fluorescence intensity observed in UCB1 (Fig. 5e, 5f) is due to an increase in the amount of suberin deposited per cell, and not the number of suberized cells in the endodermis.

\section{DISCUSSION}

\section{Both vacuolar sequestration and suberin deposition are implicated in high salt tolerance}

In the light of climate change based drought conditions which have led to increased soil salinity, pistachio provides an attractive model to dissect mechanisms of salinity tolerance and abiotic stress response (Ahmad and Prasad, 2012; Jazi et al., 2016; Bailey-Serres et al., 2019). While most of the studies so far have focused on the overall physiological plant responses (Picchioni et al., 1990; Ferguson et al., 2002; Karimi et al., 2009; Godfrey et al., 2019), there has been no analysis at the cellular and structural level in pistachio roots. In order to determine salinity tolerance mechanisms in pistachio, we analyzed $\mathrm{Na}^{+}$sequestration and apoplastic barrier differentiation across a root developmental gradient using fluorescence microscopy. Our study demonstrated a significant increase of vacuolar $\mathrm{Na}^{+}$sequestration and levels of suberin in the endo/exodermis in the more salinity tolerant UCB1 compared to P. integerrima, after salt treatment, suggesting that both mechanisms are utilized in tandem for salinity tolerance in pistachios.

Salinity tolerance is a complex trait that includes coordination of maximizing salt ion exclusion, regulation of salt ion transport, and ion sequestration at the cellular, tissue, and organ level (Tester and Davenport, 2003; Yang et al., 2009; Chen et al., 2011; Gupta and Huang, 2014; Chen et al., 2018). While salt ion extrusion at the plasma membrane is important in many species (Yang et al., 2009; Ji et al., 2013; Chen et al., 2018), it does not provide complete salinity resistance, as significant amounts of $\mathrm{Na}^{+}$still enter the roots. Thus, additional mechanisms must be employed to limit salt transport to the salinity sensitive leaves. Vacuolar sequestration of $\mathrm{Na}^{+}$is one such mechanism that reduces the amount of salt reaching the shoot, therefore reducing salt toxicity (Zhang and Blumwald, 2001; Gonzalez et al., 2012; Gupta and Huang, 2014; Guo et al., 2020). The dominant role of vacuolar $\mathrm{Na}^{+}$sequestration over $\mathrm{Na}^{+}$exclusion in salinity tolerance was recently demonstrated in barley. Screening of 45 barley accessions for salinity tolerance mechanisms, using a fluorescence microscopy approach, demonstrated a positive correlation of vacuolar $\mathrm{Na}^{+}$sequestration with the salinity tolerant varieties (Wu et al., 2019).

UCB1 plants showed a higher vacuolar $\mathrm{Na}^{+}$sequestration capacity compared to P. integerrima (Fig 3). It is likely that UCB1 is more efficient at vacuolar sequestration due to the increased activity of salt ion antiporters such as the NHX transporter family (Gupta and Huang, 2014; Bassil et al., 2019; Guo et al., 2020). Overexpression of NHX1 increases salt tolerance in diverse species such as wheat, rice, tomato and mung bean (Zhang and Blumwald, 2001; Moghaieb et al., 2014; Kumar et al., 2017; Zeng et al., 2018) and likely contributes to vacuolar sequestration in pistachio. Retention of $\mathrm{Na}^{+}$in the vacuole is also regulated by the control of $\mathrm{Na}^{+-}$permeable vacuolar channels, which mediate the back-leak of $\mathrm{Na}^{+}$into the cytosol (Isayenkov et al., 2010; Munns et al., 2016; Munns et al., 2020). Thus, control of expression or activity of these channels can also contribute to the increased vacuolar $\mathrm{Na}^{+}$in UCB1.

Another plausible scenario for lower $P$. integerrima vacuolar sequestration is that $P$. integerrima may be less efficient at minimizing salt ion entry, which leads to $\mathrm{Na}^{+}$levels exceeding its tolerance threshold, causing cell damage and loss of vacuolar sequestration capacity. Analyses of expression and activity of salt ion antiporters such as NHX, AKT1, and SOS1, along with other $\mathrm{Na}^{+}$-permeable channels and biotic stress response markers can help dissect the prominent pathway in UCB1 (Yang et al., 2009; Gupta and Huang, 2014; van Zelm et al., 2020).

\section{A root developmental gradient in vacuolar sequestration}

Sodium uptake, transport, and sequestration across the three examined roots zones are closely linked to their developmental stages. Thus, we have proposed a model of sodium tolerance based on the developmental 
gradient of pistachio roots (Fig. 6). Sodium uptake and transport at the meristematic region are relatively unhindered by apoplastic barriers, and it is accompanied by vacuole sequestration to minimize the impact of the salt ions. In the second developmental zone (zone 1), the peak of $\mathrm{Na}^{+}$sequestration in parenchyma cells likely reflects their higher vacuole storage capacity and maturation status compared to the meristematic region. This corroborates the pattern of $\mathrm{Na}^{+}$vacuolar sequestration in diverse species (Bojórquez-Quintal et al., 2014; Wu et al., 2015; Wu et al., 2019). It is possible that more ionic uptake occurs in this zone, due to the increase in the number of mature vessel elements compared to the younger, more meristematic zone 0 (Baum et al., 2002; Wachsman et al., 2015), and the higher rate of sodium entry becomes reflected in the vacuolar sequestration. Apoplastic entry of sodium to the third developmental zone (zone 2) may be more tightly controlled due to the extensive suberization of apoplastic barriers, resulting in enhanced blockage of apoplastic transport and absorption (Serra et al., 2009; Ranathunge et al., 2011; Barberon, 2017). This may lead to a reduction in the rate of sodium entry into root at zone 2 and reduced vacuolar sequestration compared to the younger developmental zones.

Together, our results suggest that vacuolar sequestration is coordinated across different developmental zones. The responses of other subcellular compartments, such as the different endosomes/vesicles beyond the vacuole, and cell type specificity (Oh et al., 2015) are added layers of complexity that can be considered within the root developmental gradient in future studies.

\section{Apoplastic barriers as a line of defense}

UCB1 shows significantly higher levels of suberin than $P$. integerrima in both the endodermis and exodermis before salt treatment, and this difference was maintained after treatment (Fig. 5b-f). Furthermore, we detected a lower concentration of $\mathrm{Na}^{+}$in UCB1 roots compared to P. integerrima (Supp. Fig. 5), a pattern that was observed earlier in shoot tissue (Ferguson et al., 2002; Godfrey et al., 2019). One can conclude that UCB1 is more efficient at minimizing salt ion entry compared to P. integerrima, and that the high $\mathrm{Na}^{+}$concentration in $P$. integerrima roots is a result of higher amounts of un-sequestered "free" $\mathrm{Na}^{+}$. Since the endodermis plays a role in the regulation of salt ion uptake by minimization of $\mathrm{Na}^{+}$entry (Chen et al., 2018), and that salinity stress can induce suberin deposition (Enstone et al., 2003; Chen et al., 2011; Byrt et al., 2018; Chen et al., 2018), the superior salinity tolerance of UCB1 may be a result of the increased suberization at the apoplastic barriers. Higher baseline level of suberization can help reduce the exposure of root cells to ion toxicity at the initiation of salt treatment, increasing the overall health of the root cells during the critical early stages of salinity stress response. Such mechanisms have been proposed for rice and citrus (Ruiz et al., 2016; Vishal et al., 2019), and a similar mechanism may be taking place in UCB1.

The increase of suberin at the cellular barriers upon salinity stress was more pronounced in the endodermis and it was significantly higher in UCB1 across all developmental zones (Fig. 5b, e, f). Interestingly, the more sensitive genotype $P$. integerrima showed an increased number of suberized cells (Fig. 5g), but not increased suberin fluorescence intensity (across the entire exodermis/endodermis), suggesting that the initiation of the cellular barriers starts earlier in P. integerrima, but its rate of suberin biosynthesis and deposition is lower than UCB1. It is possible that some $P$. integerrima root cell types mature earlier compared to UCB1, and as a result, UCB1 maintains higher root plasticity in the same developmental zone. This is also a potential explanation for the increased number of lateral roots we observed in UCB1 compared to $P$. integerrima, since patterning of lateral root primordia is known to occur at the root tip and is highly responsive to abiotic stress (Koevoets et al., 2016; Julkowska et al., 2017; Dinneny, 2019).

Salinity tolerance and suberin deposition are energetically expensive for plants, and the plant must choose between the cost of cellular barrier enforcement and the cost of salinity stress (Tyerman et al., 2019). The increased basal level in suberin deposition at the endodermis in UCB1 suggests an early energy investment in enforcing salt exclusion from the vascular cylinder, and that this mechanism is more strongly utilized by UCB1 under salinity stress. Selection pressure for crop varieties with increased apoplastic barriers suberization has been shown for barley (Kreszies et al., 2020), suggesting that this energy investment is preferred in monocots. The popularity of UCB1 in pistachio breeding indicates a similar trend is taking place in woody perennial nut crops. 


\section{Developmental gradient in suberization}

An analysis of the developmental zones showed that the increase in endodermis suberization after salt treatment in UCB1 can be mainly attributed to zone 0 and zone1 (Fig. 5d). Furthermore, in both apoplastic barriers, the greatest difference in suberization between the two genotypes, when under salinity stress, was observed in the youngest zone (Fig. 5f). The development of the apoplastic barriers is highly responsive to abiotic stress (Enstone et al., 2003; Barberon, 2017; Byrt et al., 2018; Chen et al., 2018; Kreszies et al., 2019; Wang et al., 2020), and contributes in salinity tolerance (Chen et al., 2018; Wang et al., 2020). The root meristematic zone, in particular, represents a region with the highest plasticity (Rost, 2011) that differentiates earlier under abiotic stress (Ji et al., 2014; Cajero-Sanchez et al., 2019). Our result suggests that both the timing of apoplastic barrier differentiation and the increase of suberin deposition contributed to the increase in suberization under salt stress. The development of the apoplastic barrier closer to the root tip in response to salt and other abiotic stresses in maize, barley, and rice support this hypothesis (Shen et al., 2015; Kreszies et al., 2019; Vishal et al., 2019). However, the increase in suberin deposition, rather than earlier differentiation of the apoplastic barrier, appears to be the main contributor to salinity tolerance in UCB1 when compared to P. integerrima. Salinity stress induces modifications in the overall cell wall, including suberin, lignin, and polysaccharide deposition (Byrt et al., 2018; Rui and Dinneny, 2020; Wang et al., 2020). It remains to be seen if the overall regulation of polysaccharide biosynthesis and deposition also changes in pistachio to reinforce minimization of salt ion entry and to limit toxic ion transport to leaves (Byrt et al., 2018).

\section{Conclusion}

Based on our data and the extended literature, we put forward a model describing aspects of salinity tolerance in pistachio with respect to root developmental gradient. Pistachio roots contain both endodermis and exodermis as apoplastic barriers (Fig. 6). During salinity stress, enhanced suberin deposition at these barriers, particularly in the endodermis, and vacuolar sequestration both are associated with the high salinity tolerance genotype.

This is a foundational study for the examination of pistachio root plasticity using fluorescence confocal microscopy across a fine root developmental gradient. Together, our study illustrates the importance of including different stages of development in evaluating stress tolerance mechanisms. It also suggests that both development and cell type specificity is another layer of complexity that should be taken into account in salinity stress response.

The data presented here provide a model to be considered for future investigation of the cellular mechanisms of salinity tolerance, with additional potential application for analyzing within species variation of salinity tolerance for plant breeding purposes. Woody perennial nut species, such as pistachio, provide useful examples of natural adaptation in abiotic stress that can be further utilized for a mechanistic understanding of this process. With the anticipated availability of the pistachio rootstock genomes, the aforementioned mechanisms can be uncovered at a molecular level in future studies.

\section{Acknowledgements}

We would like to thank M. R. Rosquete for his critical comments and suggestions. This study was supported by the Pistachio Board of CA HC-2017-22, HC-2019-21, and the U.S. Department of Agriculture award CA-D-PLS-2132-H to G.D.

\section{Author contributions}

SZ, AQ, TW, LF, JJ, GD conceived research. SZ, AQ, OKB, SM, ASH, GD, TW, TL performed research. SZ, AQ, OKB, SM, ASH, YS, TC, JJ, TW, GD analyzed data. SZ and GD wrote the manuscript. All the authors edited and accepted the final version of the manuscript.

\section{REFERENCES}


Ahmad P, Prasad M (2012) Abiotic stress responses in plants. Abiotic Stress New Res. doi: 10.1007/9781-4614-0634-1

Ahmad R, Anjum MA (2020) Physiological and molecular basis of salinity tolerance in fruit crops. Fruit Crop. doi: 10.1016/b978-0-12-818732-6.00032-0

Ahmad R, Ferguson L, Southwick SM (2005) Molecular marker analyses of pistachio rootstocks by Simple Sequence Repeats and Sequence-Related Amplified Polymorphisms. J Hortic Sci Biotechnol80 : 382386

Akbari M, Mahna N, Ramesh K, Bandehagh A, Mazzuca S (2018) Ion homeostasis, osmoregulation, and physiological changes in the roots and leaves of pistachio rootstocks in response to salinity. Protoplasma255 : 1349-1362

Bailey-Serres J, Parker JE, Ainsworth EA, Oldroyd GED, Schroeder JI (2019) Genetic strategies for improving crop yields. Nature575 : 109-118

Barberon M (2017) The endodermis as a checkpoint for nutrients. New Phytol 213 : 1604-1610

Bassil E, Zhang S, Gong H, Tajima H, Blumwald E (2019) Cation specificity of vacuolar NHX-type cation/H + Antiporters 1[OPEN]. Plant Physiol 179 : 616-629

Baum SF, Dubrovsky JG, Rost TL (2002) Apical organization and maturation of the cortex and vascular cylinder in Arabidopsis thaliana (Brassicaceae) roots. Am J Bot 89 : 908-920

Bernstein N, Meiri A, Zilberstaine M (2004) Root Growth of Avocado is More Sensitive to Salinity than Shoot Growth. J Am Soc Hortic Sci 129 : 188-192

Bojórquez-Quintal E, Velarde-Buendía A, Ku-González Á, Carillo-Pech M, Ortega-Camacho D, Echevarría-Machado I, Pottosin I, Martínez-Estévez M (2014) Mechanisms of salt tolerance in habanero pepper plants (Capsicum chinense Jacq.): Proline accumulation, ions dynamics and sodium root-shoot partition and compartmentation. Front Plant Sci 5 : 1-14

Byrt CS, Munns R, Burton RA, Gilliham M, Wege S (2018) Root cell wall solutions for crop plants in saline soils. Plant Sci269 : 47-55

Cajero-Sanchez W, Aceves-Garcia P, Fernández-Marcos M, Gutiérrez C, Rosas U, GarcíaPonce B, Álvarez-Buylla ER, Sánchez M de la P, Garay-Arroyo A (2019) Natural root cellular variation in responses to osmotic stress in arabidopsis thaliana accessions. Genes (Basel)10 : 1-23

Chen M, Yang Z, Liu J, Zhu T, Wei X, Fan H, Wang B (2018) Adaptation mechanism of salt excluders under saline conditions and its applications. Int J Mol Sci. doi: 10.3390/ijms19113668

Chen T, Cai X, Wu X, Karahara I, Schreiber L, Lin J (2011) Casparian strip development and its potential function in salt tolerance. Plant Signal Behav 6 : 1499-1502

Dinneny JR (2019) Developmental Responses to Water and Salinity in Root Systems. Annu Rev Cell Dev Biol 35 : 239-257

Doblas VG, Geldner N, Barberon M (2017) The endodermis, a tightly controlled barrier for nutrients. Curr Opin Plant Biol39 : 136-143

Enstone DE, Peterson CA, Ma F (2003) Root Endodermis and Exodermis : Structure, Function, and Responses to the Environment. 335-351

Ferguson L, Poss JA, Grattan SR, Grieve CM, Wang D, Wilson C, Donovan TJ, Chao C-T (2002) Pistachio Rootstocks Influence Scion Growth and Ion Relations under Salinity and Boron Stress. J Am Soc Hortic Sci127 : 194-199 
Godfrey JM, Ferguson L, Sanden B, Tixier A, Sperling O, Grattan SR, Zwieniecki MA (2019) Sodium interception by xylem parenchyma and chloride recirculation in phloem may augment exclusion in the salt tolerant Pistacia genus: context for salinity studies on tree crops. Tree Physiol 1-15

Gonzalez P, Syvertsen JP, Etxeberria E (2012) Sodium distribution in salt-stressed citrus rootstock seedlings. HortScience47 : 1504-1511

Guo Q, Tian XX, Mao PC, Meng L (2020) Overexpression of Iris lactea tonoplast Na+/H+ antiporter gene IlNHX confers improved salt tolerance in tobacco. Biol Plant 64 : 50-57

Gupta B, Huang B (2014) Mechanism of salinity tolerance in plants: Physiological, biochemical, and molecular characterization. Int J Genomics. doi: 10.1155/2014/701596

Henderson SW, Dunlevy JD, Wu Y, Blackmore DH, Walker RR, Edwards EJ, Gilliham M, Walker AR (2018) Functional differences in transport properties of natural HKT1;1 variants influence shoot $\mathrm{Na}+$ exclusion in grapevine rootstocks. New Phytol 217 : 1113-1127

Holtz B, Ferguson L, Parfitt D, Allen G, Radoicich R (2005) Rootstock production and budding. Pist Prod Man 74-79

Isayenkov S, Isner JC, Maathuis FJM (2010) Vacuolar ion channels: Roles in plant nutrition and signalling. FEBS Lett584 : 1982-1988

Jamshidi Goharrizi K, Amirmahani F, Salehi F (2020) Assessment of changes in physiological and biochemical traits in four pistachio rootstocks under drought, salinity and drought + salinity stresses. Physiol Plant 168 : 973-989

Jazi MM, Khorzoghi EG, Botanga C, Seyedi SM (2016) Identification of Reference Genes for Quantitative Gene Expression Studies in a Non-Model Tree Pistachio (Pistacia vera L.). PLoS One11 : 1-16

Ji H, Liu L, Li K, Xie Q, Wang Z, Zhao X, Li X (2014) PEG-mediated osmotic stress induces premature differentiation of the root apical meristem and outgrowth of lateral roots in wheat. J Exp Bot65 : 4863-4872

Ji H, Pardo JM, Batelli G, Van Oosten MJ, Bressan RA, Li X(2013) The salt overly sensitive (SOS) pathway: Established and emerging roles. Mol Plant 6 : 275-286

Jones K, Kim DW, Park JS, Khang CH (2016) Live-cell fluorescence imaging to investigate the dynamics of plant cell death during infection by the rice blast fungus Magnaporthe oryzae. BMC Plant Biol $16: 1-8$

Julkowska MM, Hoefsloot HCJ, Mol S, Feron R, De Boer GJ, Haring MA, Testerink C (2014) Capturing arabidopsis root architecture dynamics with root-fit reveals diversity in responses to salinity. Plant Physiol166 : 1387-1402

Julkowska MM, Koevoets IT, Mol S, Hoefsloot H, Feron R, Tester MA, Keurentjes JJB, Korte A, Haring MA, De Boer GJ, et al (2017) Genetic components of root architecture remodeling in response to salt stress. Plant Cell 29 : 3198-3213

Karimi S, Rahemi M, Maftoun M, Eshghi, Tavallali V (2009) Effects of long-term salinity on growth and performance of two pistachio (Pistacia L.) rootstocks. Aust J Basic Appl Sci 3 : 1630-1639

Koevoets IT, Venema JH, Elzenga JTM, Testerink C (2016) Roots Withstanding their Environment : Exploiting Root System Architecture Responses to Abiotic Stress to Improve Crop Tolerance. 7 : 1-19

Kreszies T, Eggels S, Kreszies V, Osthoff A, Shellakkutti N, Baldauf JA, Zeisler-Diehl V V., Hochholdinger F, Ranathunge K, Schreiber L (2020) Seminal roots of wild and cultivated barley differentially respond to osmotic stress in gene expression, suberization, and hydraulic conductivity. Plant Cell Environ 43 : 344-357 
Kreszies T, Shellakkutti N, Osthoff A, Yu P, Baldauf JA, Zeisler-Diehl V V., Ranathunge K, Hochholdinger F, Schreiber L (2019) Osmotic stress enhances suberization of apoplastic barriers in barley seminal roots: analysis of chemical, transcriptomic and physiological responses. New Phytol 221 : 180-194

Kumar S, Kalita A, Srivastava R, Sahoo L (2017) Co-expression of arabidopsis NHX1 and bar improves the tolerance to salinity, oxidative stress, and herbicide in transgenic mungbean. Front Plant Sci8 : 1-18

Lee Y, Rubio MC, Alassimone J, Geldner N (2013) A mechanism for localized lignin deposition in the endodermis. Cell 153 : 402-412

Lux A, Morita S, Abe J, Ito K (2005) An improved method for clearing and staining free-hand sections and whole-mount samples. Ann Bot 96 : 989-996

McCully M (1995) How do real roots work? Some new views of root structure. Plant Physiol 109 : 1-6

McCully ME (1999) ROOTS IN SOIL: Unearthing the Complexities of Roots and Their Rhizospheres. Annu Rev Plant Physiol Plant Mol Biol50 : 695-718

Moghaieb REA, Sharaf AN, Soliman MH, El-Arabi NI, Momtaz OA(2014) An efficient and reproducible protocol for the production of salt tolerant transgenic wheat plants expressing the Arabidopsis AtNHX1 gene. GM Crops Food 5 : 132-138

Mohammadkhani N, Heidari R, Abbaspour N, Rahmani F (2016) Salinity effects on expression of some important genes in sensitive and tolerant grape genotypes. Turkish J Biol 40 : 95-108

Munns R, James RA, Gilliham M, Flowers TJ, Colmer TD (2016) Tissue tolerance: an essential but elusive trait for salt-tolerant crops. Funct Plant Biol 43 : 1103-1113

Munns R, Passioura JB, Colmer TD, Byrt CS (2020) Osmotic adjustment and energy limitations to plant growth in saline soil. New Phytol 225 : 1091-1096

Naseer S, Lee Y, Lapierre C, Franke R, Nawrath C, Geldner N(2012) Casparian strip diffusion barrier in Arabidopsis is made of a lignin polymer without suberin. Proc Natl Acad Sci U S A 109 : 10101-10106

Oh DH, Barkla BJ, Vera-Estrella R, Pantoja O, Lee SY, Bohnert HJ, Dassanayake M (2015) Cell type-specific responses to salinity - the epidermal bladder cell transcriptome of Mesembryanthemum crystallinum. New Phytol 207 : 627-644

Park M, Lee H, Lee JS, Byun MO, Kim BG (2009) In planta measurements of Na+ using fluorescent dye CoroNa Green. J Plant Biol52 : 298-302

Picchioni GA, Miyamoto S, Storey JB (1990) Salt Effects on Growth and Ion Uptake of Pistachio Rootstock Seedlings. J Am Soc Hortic Sci 115 : 647-653

Pradhan Mitra P, Loque D (2014) Histochemical staining of Arabidopsis thaliana secondary cell wall elements. J Vis Exp 1-11

R Core Team (2017) R: A language and environment for statistical computing. R Foundation for Statistical Computing. Vienna, Austria, https://www.r-project.org/.

Rahneshan Z, Nasibi F, Moghadam AA (2018) Effects of salinity stress on some growth, physiological, biochemical parameters and nutrients in two pistachio (Pistacia vera L.) rootstocks. J Plant Interact 13 : 73-82

Ramirez-Flores MR, Rellan-Alvarez R, Wozniak B, Gebreselassie MN, Jakobsen I, OlaldePortugal V, Baxter I, Paszkowski U, Sawers RJH(2017) Co-ordinated changes in the accumulation of metal ions in maize (Zea mays ssp. mays L.) in response to inoculation with the arbuscular mycorrhizal fungus funneliformis mosseae. Plant Cell Physiol58 : 1689-1699 
Ranathunge K, Schreiber L, Franke R (2011) Suberin research in the genomics era-New interest for an old polymer. Plant Sci180 : 399-413

Rewald B, Raveh E, Gendler T, Ephrath JE, Rachmilevitch S(2012) Phenotypic plasticity and water flux rates of Citrus root orders under salinity. J Exp Bot 63 : 2717-2727

Rosquete MR, Worden N, Ren G, Sinclair RM, Pfleger S, Salemi M, Phinney BS, Domozych D, Wilkop T, Drakakaki G (2019) AtTRAPPC11/ROG2: A role for TRAPPs in maintenance of the plant trans-golgi network/early endosome organization and function. Plant Cell 31 : 1879-1898

Rost TL (2011) The organization of roots of dicotyledonous plants and the positions of control points. Ann Bot 107 : 1213-1222

Rui Y, Dinneny JR (2020) A wall with integrity: surveillance and maintenance of the plant cell wall under stress. New Phytol225 : 1428-1439

Ruiz M, Quinones A, Martinez-Cuenca MR, Aleza P, Morillon R, Navarro L, Primo-Millo E, Martinez-Alcantara B (2016) Tetraploidy enhances the ability to exclude chloride from leaves in carrizo citrange seedlings. J Plant Physiol 205 : 1-10

Schneider CA, Rasband WS, Eliceiri KW (2012) NIH Image to ImageJ: 25 years of image analysis. Nat Methods 9 : 671-675

Serra O, Soler M, Hohn C, Sauveplane V, Pinot F, Franke R, Schreiber L, Prat S, Molinas M, Figueras M (2009) CYP86A33-targeted gene silencing in potato tuber alters suberin composition, distorts suberin lamellae, and impairs the periderm's water barrier function. Plant Physiol 149 : 1050-1060

Shen J, Xu G, Zheng HQ (2015) Apoplastic barrier development and water transport in Zea mays seedling roots under salt and osmotic stresses. Protoplasma 252 : 173-180

Shi H, Quintero FJ, Pardo JM, Zhu JK (2002) The putative plasma membrane NA+/H+ antiporter SOS1 controls long-distance NA+ transport in plants. Plant Cell 14:465-477

Storey R, Walker RR (1998) Citrus and salinity. Sci Hortic (Amsterdam) 78:39-81

Tataranni G, Santarcangelo M, Sofo A, Xiloyannis C, Tyerman SD, Dichio B (2015) Correlations between morpho-anatomical changes and radial hydraulic conductivity in roots of olive trees under water deficit and rewatering. Tree Physiol 35 : 1356-1365

Tester M, Davenport R (2003) Na+ tolerance and Na+ transport in higher plants. Ann Bot 91 : 503-527

Tyerman SD, Munns R, Fricke W, Arsova B, Barkla BJ, Bose J, Bramley H, Byrt C, Chen Z, Colmer TD, et al (2019) Energy costs of salinity tolerance in crop plants. New Phytol 221 : 25-29

Vishal B, Krishnamurthy P, Ramamoorthy R, Kumar PP (2019) OsTPS8 controls yield-related traits and confers salt stress tolerance in rice by enhancing suberin deposition. New Phytol 221 : 1369-1386

Wachsman G, Sparks E, Benfey PN (2015) Genes and networks regulating root anatomy and architecture Tansley review Genes and networks regulating root anatomy and architecture. 26-38

Walker RR, Torokfalvy E, Behboudian MH (1987) Uptake and distribution of chloride, sodium and potassium ions and growth of salt-treated pistachio plants. Aust J Agric Res 38 : 383-394

Wang P, Wang CM, Gao L, Cui YN, Yang HL, de Silva NDG, Ma Q, Bao AK, Flowers TJ, Rowland O, et al (2020) Aliphatic suberin confers salt tolerance to Arabidopsis by limiting $\mathrm{Na}+$ influx, $\mathrm{K}+$ efflux and water backflow. Plant Soil 448 : 603-620

Wu H, Shabala L, Liu X, Azzarello E, Zhou M, Pandolfi C, Chen ZH, Bose J, Mancuso S, Shabala S (2015) Linking salinity stress tolerance with tissue-specific Na+ sequestration in wheat roots. Front Plant Sci 6 : $1-13$ 
Wu H, Shabala L, Zhou M, Su N, Wu Q, Ul-Haq T, Zhu J, Mancuso S, Azzarello E, Shabala S (2019) Root vacuolar $\mathrm{Na}+$ sequestration but not exclusion from uptake correlates with barley salt tolerance. Plant J100 : 55-67

Yang Q, Chen ZZ, Zhou XF, Yin HB, Li X, Xin XF, Hong XH, Zhu JK, Gong Z (2009) Overexpression of SOS (salt overly sensitive) genes increases salt tolerance in transgenic Arabidopsis. Mol Plant2 : 22-31

Yang Y, Guo Y (2018) Elucidating the molecular mechanisms mediating plant salt-stress responses. New Phytol 217 : 523-539

Yeo AR, Flowers SA, Rao G, Welfare K, Senanayake N, Flowers TJ(1999) Silicon reduces sodium uptake in rice (Oryza sativa L.) in saline conditions and this is accounted for by a reduction in the transpirational bypass flow. Plant, Cell Environ 22 : 559-565

van Zelm E, Zhang Y, Testerink C (2020) Salt Tolerance Mechanisms of Plants. Annu Rev Plant Biol $71: 403-433$

Zeng Y, Li Q, Wang H, Zhang J, Du J, Feng H, Blumwald E, Yu L, Xu G (2018) Two NHXtype transporters from Helianthus tuberosus improve the tolerance of rice to salinity and nutrient deficiency stress. Plant Biotechnol J 16 : 310-321

Zhang HX, Blumwald E (2001) Transgenic salt-tolerant tomato plants accumulate salt in foliage but not in fruit. Nat Biotechnol19 : 765-768

Zhang J, Wang L, Liu Y, Li D, Feng S, Yang J, Zhang J, Wang D, Gan Y (2019) Improving salt tolerance in potato through overexpression of AtHKT1 gene. BMC Plant Biol 19 : 1-15

Zohary D, Spiegl-Roy P (1975) Beginning of fruit growing in the old world. Science (80- ) 187 : 319-327

Figure legends

Figure 1. More UCB1 seedlings exhibited high tolerance phenotype compared to $P$. integerrima

(a) Representative high and low salinity tolerance phenotypes for the paternal genotype P. integerrima and hybrid line UCB1 after one week $100 \mathrm{mM} \mathrm{NaCl}$ treatment.

(b) Percentage of individuals in each genotype showing high, moderate, or low tolerance phenotypes. N=15 for P. integerrima, 22 for UCB1.

Figure 2. Short term salt treatment did not significantly reduce the growth of UCB1 or P. integerrima .

(a) Quantification of fresh weight after one week salt treatment showed that UCB1 seedlings were comparable in fresh weight to $P$. integerrima, and neither showed significant decreases with salt treatment in either total or tissue specific weight (NS, two-way ANOVA, N=7-9 plants per treatment per genotype).

(b) UCB1 exhibited a significantly higher number of lateral roots (LRs) per plant compared to P. integerrima $(\mathrm{P}<0.05$ between genotypes, two-way ANOVA). This difference was increased after salt treatment $(\mathrm{P}<0.01$ between treatment, two-way ANOVA, $\mathrm{P}=0.01$, Tukey's HSD, $\mathrm{N}=16-17$ per treatment per genotype). Error bars $=$ SEM.

Figure 3. UCB1 showed increased vacuolar sodium sequestration compared to P. integerrima .

(a) UCB1 and $P$. integerrima root tip section stained for $\mathrm{Na}^{+}$showed increased number of vacuoles in UCB1 (indicated by arrowheads) after salt treatment and accumulation of $\mathrm{Na}^{+}$at the exodermis and endodermis (3D construction was shown for zone 1 salt treated $\mathrm{UCB} 1$ to better illustrate $\mathrm{Na}^{+}$vacuole localization).

(b) Diagram of root tip zones staged by xylem development. $\mathrm{z} 0=$ zone $0, \mathrm{z} 1=$ zone $1, \mathrm{z} 2=$ zone 2 .

(c) Quantification of average number of vacuoles per cross section. ( $\mathrm{P}<0.01$ between genotype, $\mathrm{P}<0.05$ between treatment, two-way ANOVA; ${ }^{*} \mathrm{P}=0.05$ Tukey's HSD.) (d) Quantification of average number of $\mathrm{Na}^{+}$ 
vacuoles staged by xylem development showed the highest vacuole count in zone 1 (NS, two-way ANOVA, $\mathrm{N}=15-51$ sections/zone/genotype from 3-5 plants). Error bars = SEM.

Figure 4. Sodium staining in UCB1 showed co-localization with a vacuolar marker.

(a-c) Co-staining of $\mathrm{Na}^{+}$with CoroNa green (a) and SNARF-1 for vacuole (b) showed co-localization in the vacuoles of salt-treated UCB1 root cross section (c, indicated by arrow). CoroNa Green staining alone (d-f), or SNARF-1 alone (g-i) do not show vacuolar co-localization.

Figure 5. UCB1 showed higher suberin deposition than $P$. integerrima in control and salt treated plants.

(a) Cross sections of root tips in both UCB1 and P. integerrimashowed increased suberization at exodermis (white arrow) and endodermis (red arrow) when stained with Fluorol Yellow 088. (b) Quantification of suberin fluorescence intensity (a. u.) across cross sections indicated increase in suberization in response to salt treatment in both exodermis and endodermis of P. integerrima and in endodermis of UCB1.

(c-d) Quantification of suberin fluorescence in zones staged by xylem development showed a peak in suberin increase after salt treatment in zone 1 of $P$. integerrima exodermis. In UCB1 both zone 0 and zone 1 showed an increase, with the greatest change occurring in endodermis of zone 0 .

(e-f) UCB1 showed consistently higher suberization than $P$. integerrima in the exodermis of untreated plants for all zones, and higher suberization in zone 0 of endodermis. After salt treatment, UCB1 showed higher suberization than $P$. integerrima for all zones in both exodermis and endodermis.

(g) Salt treatment increased the percentage of suberized cells in the endodermis of $P$. integerrima and in both the exodermis and endodermis of UCB1 ( $* \mathrm{P}<0.05$, ${ }^{* *} \mathrm{P}<0.01$, unpaired 2-tailed t-test) ( $\mathrm{N}=28-62$ sections collected from 3-5 plants for B, G. N=3-28 sections per zone per genotype per treatment for C-F). Error bars $=$ SEM.

Figure 6 . Model of UCB1 and P. integerrima root tip response to short-term salt treatment.

Under salinity stress, UCB1 plants were able to sequester excess $\mathrm{Na}^{+}$in the vacuoles of cortical parenchyma of the root tips in contrast to $P$. integerrima. Under control conditions UCB1 deposited more suberin in cellular barriers. In addition, while both UCB1 and $P$. integerrima showed increased suberization of endodermis and exodermis in response to salt treatment, UCB1 showed higher suberization than P. integerrima. 

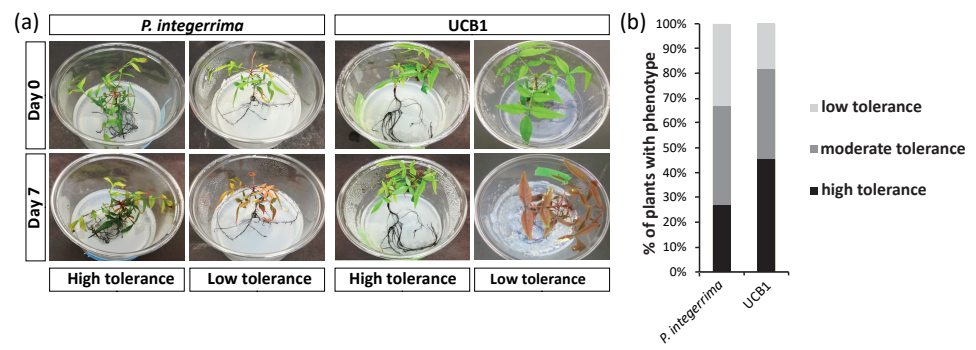

Figure 1. More UCB1 seedlings exhibited high tolerance phenotype compared to P. integerrim

(a) Representative high and low salinity tolerance phenotypes for the paternal genotype $P$. integerrima and hybrid line UCB1 after one

week $100 \mathrm{mM}$ NaCl Ireatment.
(b) Percentage of individuals in each genotype showing high, moderate, or low tolerance phenotypes. $\mathrm{N}=15$ for $P$. integerrima, 22 for 


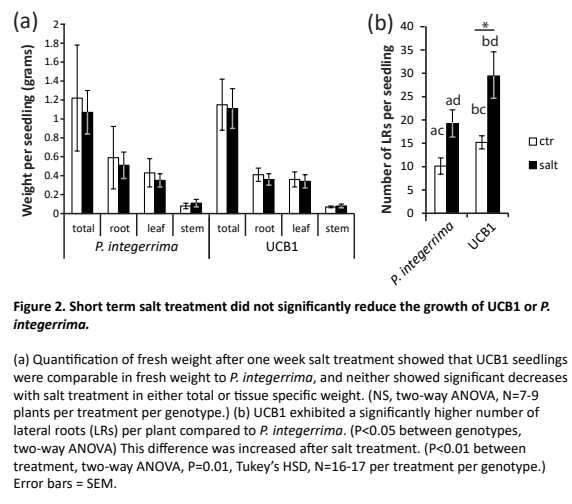



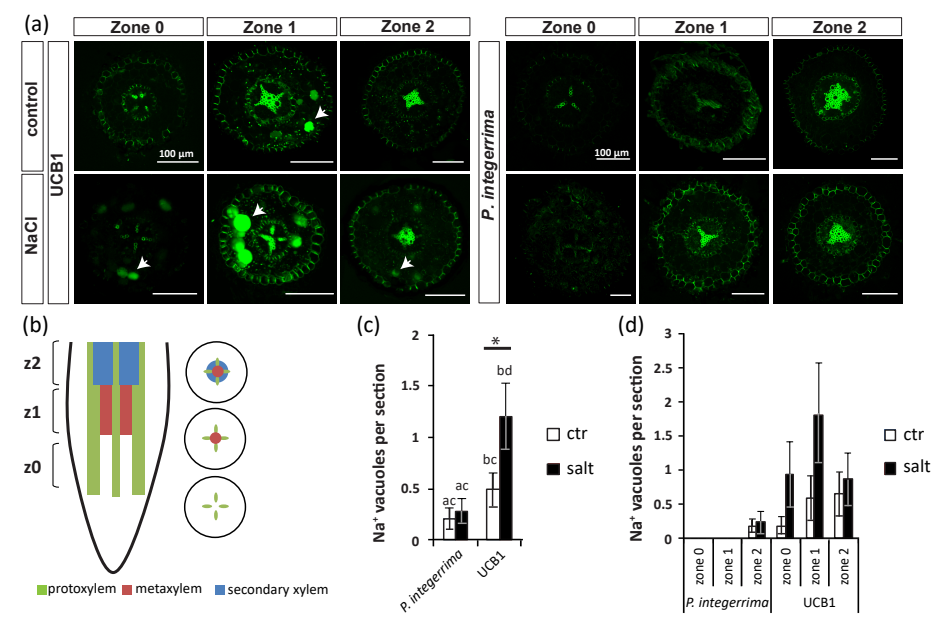

Figure 3. UCB1 showed increased vacuolar sodium sequestration compared to $P$. integerrima.

(a) UCB1 and P. integerrima root tip section stained for Na+ showed increased number of vacuoles in UCB1 (indicated by arrowheads) after salt treatment and accumulation of $\mathrm{Na}+$ at the exodermis and endodermis $(3 \mathrm{D}$ construction was shown for zone 1 salt treated UCB1 to better illustrate

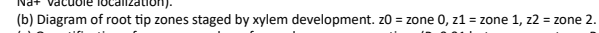

(c) Quantifiction of a $P=0.05$ Tukey's HSD.) (d) Quantification of average number of Na+ vacuoles staged by xylem development showed the highest vacuole count in zone 1 (NS, two-way ANOVA, N=15-51 sections/zone/genotype from 3-5 plants). Error bars = SEM. 


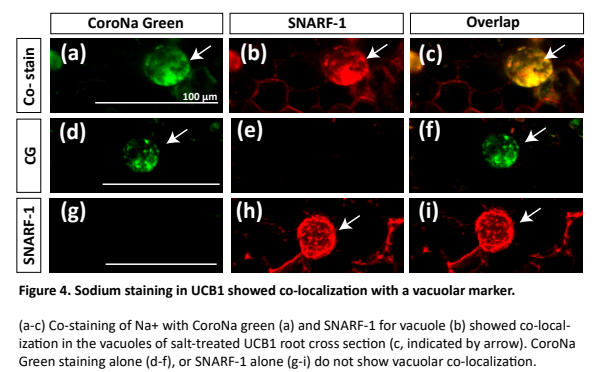



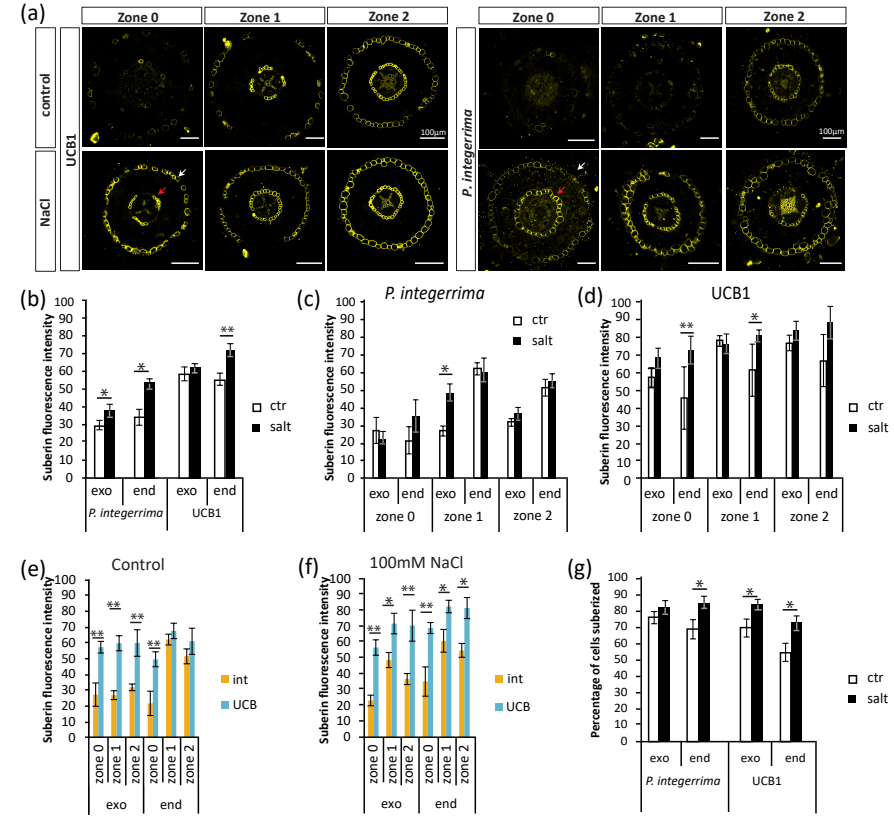

Figure 5. UCB1 showed higher suberin deposition than P. integerrima in control and salt treated plants.

(a) Cross sections of root tips in both UCB1 and P. integerrima showed increased suberization at exodermis (white arrow) and endodermis (red arrow) when stained with Fluorol Yellow 088. (b) Quantification of suberin fluorescence intensity (a. u.) across cross sections indicated increase in suberization in response to salt treatment in both exodermis and (c-d) Quantification of suberin fluorescence in zones staged by xylem development showed a peak in suberin increase after salt treatment in zone 1 of $P$. integerrima exodermis. In (c-d) Quantifcation of suberin fluorescence in zones staged by xylem development showed a peak in suberin increase ater sat both zone 0 and zone 1 showed an increase, with the greatest change occurring in endodermis of zone 0 .
UCB salt treatment, UCB1 showed higher suberization than P. integerrima for ail zones in both exodermis and endodermis. (g) Salt treatment increased the percentage of suberized cells in the endodermis of 


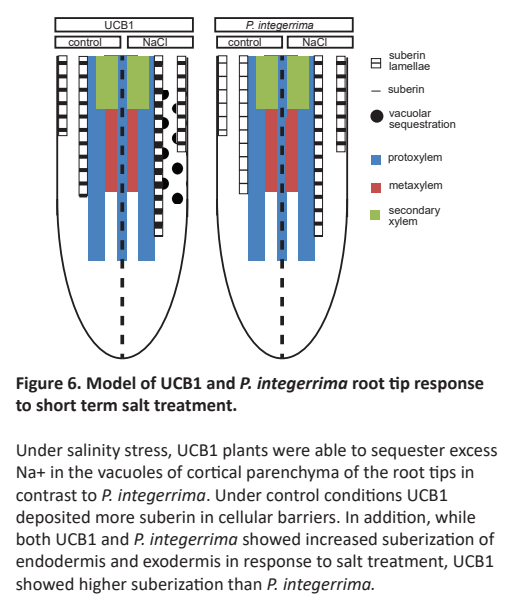

